\title{
Termografia Infravermelha no Desenvolvimento de Produtos: formas de abordagem e categorias dos produtos
}

\author{
Infrared Thermography in Product Development: approaches and categories of \\ products
}

FORCELINI, Franciele; Mestranda em Design; Universidade Federal de Santa Catarina francieleforcelini@gmail.com

MERINO, Eugenio Andrés Díaz; Doutor em Eng. Produção; Universidade Federal de Santa Catarina eugenio.merino@ufsc.br

\section{Resumo}

Considerando a contribuição das tecnologias no processo de desenvolvimento de produtos mais eficientes e eficazes, este artigo visa analisar a aplicação da termografia infravermelha no desenvolvimento de produtos, destacando as formas de abordagem e as categorias dos produtos analisados. Caracteriza-se como um estudo teórico, qualitativo, exploratório, descritivo e se utiliza de uma revisão bibliográfica sistemática para mapear estudos nacionais e internacionais. Os resultados apontam a aplicação da termografia infravermelha em distintos momentos do desenvolvimento de produtos, como no levantamento de dados e na avaliação de alternativas. Assim, os dados objetivos gerados auxiliam na análise das relações de usabilidade e conforto, amparando a tomada de decisões. As tecnologias assistivas denotam a categoria de produtos mais explorada, seguida pela de vestuário, equipamentos esportivos, mobiliário, embalagens e ferramentas. Contudo, percebe-se a aplicação ainda limitada da termografia no desenvolvimento de produtos e a oportunidade de estudos referentes aos seus protocolos e formas de análise.

Palavras Chave: design; desenvolvimento de produtos; termografia infravermelha.

\begin{abstract}
Considering the contribution of technologies to the process of developing more efficient and effective products, this article aims to analyze the application of infrared thermography in product development, highlighting the approaches and categories of products analyzed. It is characterized as a theoretical, qualitative, exploratory, descriptive study and a systematic bibliographic review is used to map national and international studies. The results point out the application of infrared thermography at different moments of product development, such as data collection and the evaluation of alternatives. Thus, the objective data generated helps in the analysis of usability and comfort relations, supporting the decision making process. Assistive technologies denote the most explored product category, followed by clothing, sports equipment, furniture, packaging and tools. However, the application of thermography in the development of products is limited and there are opportunities of studies regarding its protocols and forms of analysis.
\end{abstract}

Keywords: design; product development; infrared thermography. 


\section{Introdução}

O design de produtos tem como finalidade a geração e o desenvolvimento de soluções eficientes e eficazes por meio de um processo que leva a novos produtos (MORRIS, 2010) e envolve a concepção, a elaboração e o desenvolvimento dos projetos (GOMES FILHO, 2006). De modo geral, caracteriza-se como um processo criativo e de resolução de problemas, que inicia com a definição de um problema, passa pela coleta de informações para o estabelecimento de análises e relações, pela geração de alternativas e, por fim, chega ao desenvolvimento da solução mais adequada, que satisfaça as necessidades dos usuários (LÖBACH, 2001).

Este processo refere-se à execução de um conjunto de ações e etapas que visam atingir progressivamente determinado resultado e, para tanto, abrange diferentes disciplinas, processos e metodologias, convenientes aos seus objetivos (BEST, 2012). A este respeito, Merino et al. (2017) e Speck et al. (2016) destacam os instrumentos tecnológicos como recursos auxiliares no desenvolvimento e avaliação de produtos, visto que permitem a obtenção de dados quantificáveis, possibilitando análises mais objetivas e confiáveis, que podem minimizar incertezas e garantir dados precisos sobre o problema ou necessidade atendida.

Neste sentido, a termografia infravermelha é um instrumento tecnológico que permite o registro da distribuição da radiação térmica emitida pela superfície de um corpo, transformando-a em valores de temperatura. Baseia-se na deteç̧ão da radiação infravermelha, a qual é convertida em uma imagem térmica ou termograma, capaz de exprimir as variações de temperatura por meio de um espectro de cores, tornando possível a sua observação (GABRIEL et al., 2016; MOBLEY, 2002; SILVA, 2017). Caracteriza-se como um método não destrutivo e sem contato direto que possibilita a obtenção de um quadro visual de temperaturas, permitindo a observação de padrões de distribuição de calor, que podem ser comparadas e analisadas por meio de softwares específicos (BRIOSCHI; MACEDO; MACEDO, 2003; HOLST, 2000).

A evolução da termografia infravermelha decorre há mais de dois séculos, porém, teve seus maiores avanços tecnológicos nas últimas décadas, devido aos sistemas de alta resolução, capazes de produzir centenas de imagens por segundo e processar a imagem em tempo real (HOLST, 2000). Estes progressos também contemplam a incorporação de novos recursos aos equipamentos de medição e o desenvolvimento de versões compactas e econômicas, que permitem ampliar e flexibilizar a sua aplicação. Deste modo, devido às suas características e vantagens, a termografia infravermelha vem sendo utilizada em diversas áreas, como a industrial, elétrica, civil (FLIR, 2016), militar, médica, petroquímica, veterinária, automotiva, bem como a de segurança, salvamento, estudos ambientais, inspeções elétricas e mecânicas, óleo e gás, metais e mineração e de eletrônicos e semicondutores (SILVA, 2017).

No entanto, no campo do design ainda são encontrados poucos estudos sobre a aplicação da termografia infravermelha no processo de desenvolvimento de produtos. Apesar disso, a técnica é apontada como um recurso auxiliar no desenvolvimento de produtos por Merino et al. (2017), Silva e Mira (2016), Speck et al. (2016) e Silva, Taralli e Melz (2015), que destacam a obtenção de dados diferenciados e objetivos, os quais podem ser utilizados pelas equipes de projeto no desenvolvimento e avaliação de produtos. Ou seja, a termografia infravermelha pode permitir uma exploração aprofundada dos produtos, auxiliando no diagnóstico do mau uso de materiais e peças, falhas de projeto, bem como de percepções dos usuários, otimizando o processo de desenvolvimento e redesign de produtos (SILVA; TARALLI; MELZ, 2015). 
Contudo, entendendo as capacidades da termografia infravermelha e suas vantagens no desenvolvimento de produtos mais eficientes, confortáveis e seguros, este artigo visa analisar a aplicação da termografia infravermelha no desenvolvimento de produtos, destacando as formas de abordagem e as categorias dos produtos analisados. Para tanto, utiliza-se de um estudo de natureza teórica, abordagem qualitativa, cunho exploratório e descritivo e utiliza, como procedimento técnico, uma revisão bibliográfica sistemática em bases de dados de teses e dissertações, artigos de periódicos e de anais de eventos, em âmbito nacional e internacional.

\section{Procedimentos Metodológicos}

Este estudo possui natureza teórica e abordagem qualitativa, com enfoque em descrições detalhadas de situações, pessoas e interações observadas e suas manifestações (SAMPIERI; COLLADO; LUCIO, 2013). Quanto aos seus objetivos, caracteriza-se como exploratório e descritivo. A etapa exploratória visa a familiarização com a temática, proporcionando uma visão geral, enquanto a descritiva pretende descrever as características dos fenômenos, para analisar como são e como se manifestam (GIL, 2008; SAMPIERI; COLLADO; LUCIO, 2013). Logo, este artigo busca mapear estudos com a aplicação da termografia infravermelha no desenvolvimento de produtos, identificando e descrevendo as formas de abordagem e as categorias dos produtos analisados.

No que tange os procedimentos técnicos, foi realizada uma revisão bibliográfica sistemática para o levantamento dos estudos com a aplicação da termografia infravermelha no desenvolvimento de produtos, possibilitando uma visão geral por meio de métodos explícitos e reprodutíveis (GREENHALGH, 1997). Este método "permite maximizar o potencial de uma busca, encontrando o maior número possível de resultados de uma maneira organizada" (KOLLER; DE PAULA COUTO; VON HOHENDORFF, 2014, p.56).

\subsection{Etapas de pesquisa}

A revisão bibliográfica sistemática foi dividida em oito etapas (figura 1), conforme proposto por Koller, De Paula Couto e Von Hohendorff (2014): (1) delimitação da questão de pesquisa, (2) escolha das fontes de dados, (3) definição das palavras-chave para busca, (4) busca e armazenamento dos resultados, (5) seleção pelo resumo, de acordo com os critérios de inclusão e exclusão; (6) extração dos dados, (7) avaliação dos materiais selecionados e, por fim, (8) síntese e interpretação dos dados sobre as abordagens e as categorias de produtos analisados nos estudos que aplicaram a termografia infravermelha no desenvolvimento de produtos.

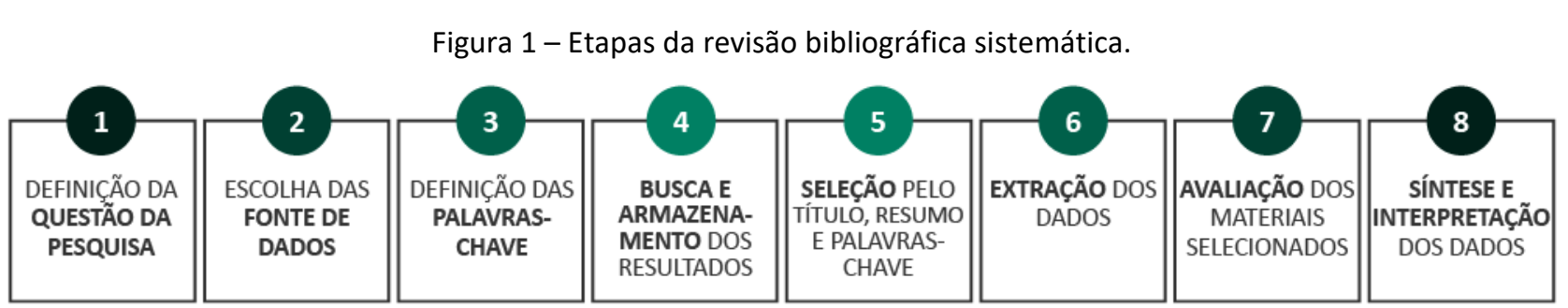

Fonte: Elaborado com base em Koller, De Paula Couto e Von Hohendorff (2014).

\subsection{Materiais e métodos}

Na etapa 1 foi delimitada a questão de pesquisa para a realização da revisão bibliográfica sistemática, que aconteceu nos meses de outubro e novembro de 2017. A etapa 2 contemplou a 
seleção das bases de dados: quatro bases de teses e dissertações, partindo do âmbito local (UFSC), nacional (CAPES e BDTD) e ao mundial (ProQuest), e em cinco bases de dados de artigos de periódicos e de anais de eventos, partindo do âmbito nacional e latino-americano (Scielo) ao mundial (Scopus, Web of Science, HBSCO e PubMed).

Na etapa 3 foram definidas as palavras-chave para a busca, utilizando-se os termos "termografia" (thermography ou termograph*) e produto (product) ou design de produto (product design*) ou desenvolvimento de produto (product develop*) ou design industrial (industrial design). O modo de busca foi adaptado aos diferentes mecanismos das bases de dados.

$\mathrm{Na}$ etapa 4, de busca e armazenamento dos resultados, foram considerados os estudos (teses, dissertações, artigos de periódicos e de anais de eventos) escritas em idiomas dominados (inglês, português e espanhol), entre os anos de 2007 e 2017, possuindo documentos completos. Os resultados foram armazenados no software EndNote, o qual auxiliou na eliminação de estudos duplicados (F1) e demais fases do processo de filtragem, conforme figura 2.

Figura 2 - Processos de filtragem da revisão bibliográfica sistemática.

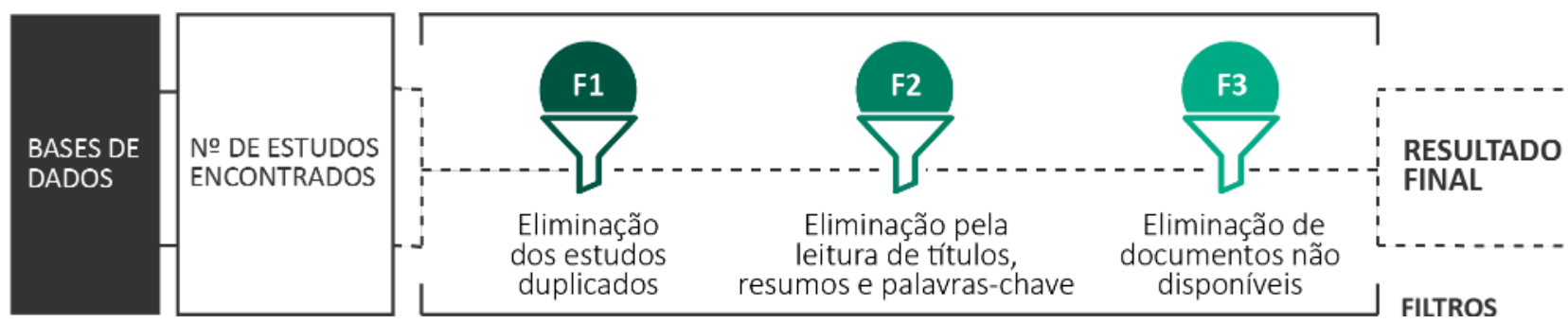

Fonte: Elaborado pela autora.

A etapa 5 contemplou a seleção dos estudos pela leitura de títulos, resumos e palavras-chave (F2) relacionadas a aplicação da termografia infravermelha na área do design/desenvolvimento de produtos (escopo), desconsiderando estudos com a aplicação da termografia infravermelha em outras áreas. Posteriormente, considerando critérios de inclusão e exclusão, foram eliminados os estudos cujos documentos não se encontravam acessíveis no portal de periódicos da CAPES, na UFSC, Google Acadêmico, sistema COMUT e com acesso que envolvesse pagamento (F3).

$\mathrm{Na}$ etapa 6 foi realizada a extração dos dados dos documentos selecionados, utilizando-se uma planilha do Microsoft Excel, dividida nos seguintes tópicos: objetivo do estudo (1), estudo com seres humanos (2), estudo com produtos (3), categoria de produto analisado (4), tipo de produto analisado (5) e tecnologias complementares (6). Esta organização permitiu a realização das etapas 7 e 8, que contemplam a avaliação dos materiais selecionados (7), a síntese e a interpretação dos dados (8), possibilitando a análise de conteúdo sobre as formas de abordagem e as categorias dos produtos analisados nos estudos com a aplicação da termografia infravermelha no desenvolvimento de produtos.

\section{Resultados}

Os resultados e os processos de filtragem dos estudos são apresentados na figura 3. A este respeito, o quadro 1 expõe os 21 estudos relacionados a aplicação da termografia infravermelha no desenvolvimento/design de produtos, sendo 12 artigos de periódicos, 7 dissertações, 1 tese e 1 artigo de evento. 
Figura 3 - Resultados e processos da revisão bibliográfica sistemática - teses, dissertações e artigos.

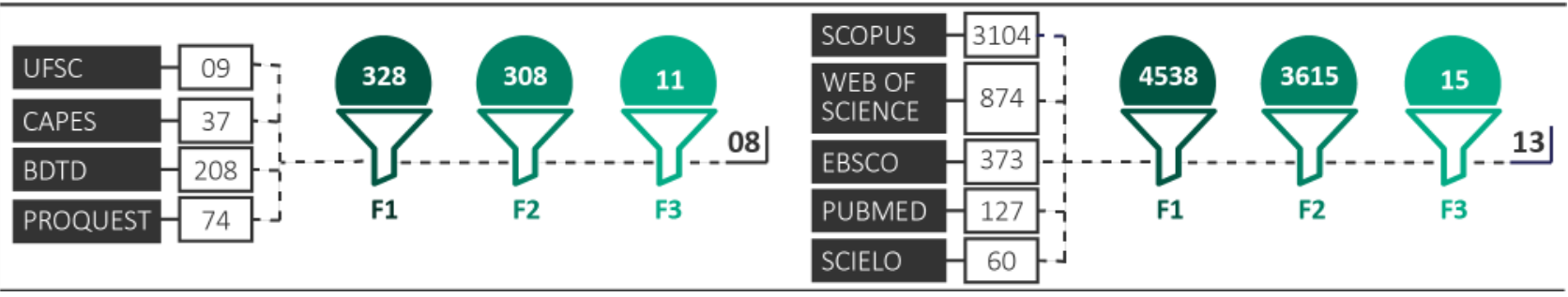

Fonte: Elaborado pela autora.

Quadro 1 - Relação de estudos selecionados (autor, país e título do trabalho).

\begin{tabular}{|c|c|c|c|}
\hline Ano & Autores & Tipo & Título \\
\hline 2017 & MERINO et al. & $\mathrm{E}$ & Implementation of integrated instrumentation in assistive technology \\
\hline 2017 & SALES et al. & $\mathrm{P}$ & Thermal comfort of seats as visualized by infrared thermography \\
\hline 2017 & TIRLONI et al. & $\mathrm{P}$ & $\begin{array}{l}\text { Thermographic evaluation of the hands of pig slaughterhouse workers exposed } \\
\text { to cold temperatures. }\end{array}$ \\
\hline 2017 & $\begin{array}{l}\text { PUŠNIK; ČUK; } \\
\text { HADŽIČ }\end{array}$ & $\mathrm{P}$ & Influence of new anatomic ring design on palm skin temperature \\
\hline 2016 & BARROS et al. & $\mathrm{P}$ & $\begin{array}{l}\text { Using digital thermography to analyse the product user's affective experience of } \\
\text { a product }\end{array}$ \\
\hline 2016 & DOTTI et al. & $\mathrm{P}$ & $\begin{array}{l}\text { Thermo-physiological comfort of soft-shell back protectors under controlled } \\
\text { environmental conditions }\end{array}$ \\
\hline 2016 & BARROS & $\mathrm{D}$ & $\begin{array}{l}\text { Aplicação da Neuroergonomia, rastreamento ocular e termografia por } \\
\text { infravermelho na avaliação de produto de consumo: um estudo de usabilidade. }\end{array}$ \\
\hline 2016 & PERAZZO & $\mathrm{D}$ & $\begin{array}{l}\text { Tecnologia Assistiva: a influência do ângulo do Tild sobre as pressões em } \\
\text { assentos de cadeiras de rodas. }\end{array}$ \\
\hline 2015 & $\begin{array}{l}\text { FLORES- } \\
\text { OLIVARES et al. }\end{array}$ & $\mathrm{P}$ & $\begin{array}{l}\text { Preliminary Study on the Evaluation of Musculoskeletal Risks through Infrared } \\
\text { Thermography for Drummers }\end{array}$ \\
\hline 2015 & SILVA et al. & $\mathrm{P}$ & Evaluation of Two PET Bottles Caps: An Exploratory Study \\
\hline 2015 & $\begin{array}{l}\text { ROSSIGNOLI; } \\
\text { BENITO; } \\
\text { HERRERO }\end{array}$ & $\mathrm{P}$ & $\begin{array}{l}\text { Reliability of infrared thermography in skin temperature evaluation of } \\
\text { wheelchair users }\end{array}$ \\
\hline 2015 & SANTOS & $\mathrm{D}$ & $\begin{array}{l}\text { Relação entre design da modelagem e aplicação de materiais de mudança de } \\
\text { fase no vestuário: uma análise do conforto térmico com base nos fatores } \\
\text { humanos. }\end{array}$ \\
\hline 2014 & ANSELMO & $\mathrm{D}$ & $\begin{array}{l}\text { Parâmetros para o desenvolvimento de sapatilhas femininas de uso diário com } \\
\text { ênfase no conforto térmico e percepção da usuária. }\end{array}$ \\
\hline 2014 & PORTO & $\mathrm{D}$ & Proposta de avaliação de conforto térmico em órteses esportivas de joelho. \\
\hline
\end{tabular}




\begin{tabular}{|c|c|c|c|}
\hline 2013 & PEREIRA & $\mathrm{D}$ & Características Térmicas de Assento de Cadeiras Escolares por Termografia. \\
\hline 2011 & SILVA et al. & $\mathrm{P}$ & $\begin{array}{l}\text { Design and milling manufacture of polyurethane custom contoured cushions for } \\
\text { wheelchair users }\end{array}$ \\
\hline 2011 & PRESTES & $\mathrm{D}$ & $\begin{array}{l}\text { Tecnologia assistiva: atributos de design de produto para adequação postural } \\
\text { personalizada na posição sentada }\end{array}$ \\
\hline 2011 & SILVA & $\mathrm{T}$ & $\begin{array}{l}\text { Usinagem de espumas de poliuretano e digitalização tridimensional para } \\
\text { fabricação de assentos personalizados para pessoas com deficiência. }\end{array}$ \\
\hline 2010 & LUZ et al. & $\mathrm{P}$ & $\begin{array}{l}\text { Adaptação à prótese híbrida de extremidade superior: estudo termográfico de } \\
\text { um caso. }\end{array}$ \\
\hline 2009 & $\begin{array}{l}\text { JENKINS; ROWN; } \\
\text { RUTTERFORD }\end{array}$ & $\mathrm{P}$ & $\begin{array}{l}\text { Comparing thermographic, EEG, and subjective measures of affective experience } \\
\text { during simulated product interactions }\end{array}$ \\
\hline 2008 & SCHACHER et al. & $\mathrm{P}$ & Improvement of dentist gowns - new constraints and new risks \\
\hline
\end{tabular}

Legenda: Artigo de periódico (P), artigo de evento (E), dissertação (D) e tese (T).

Fonte: Elaborado pela autora (2018).

Contudo, os resultados foram organizados e os dados extraídos e classificados de modo a facilitar a análise de conteúdo sobre as formas de abordagem e categorias de produtos analisados.

\section{Discussão dos resultados}

Diante dos resultados, foi possível identificar que todos os estudos contemplam experimentos termográficos com seres humanos (MERINO et al., 2017; SALES et al., 2017; TIRLONI et al., 2017; PUŠNIK; ČUK; HADŽIČ, 2017; BARROS et al., 2016; DOTTI et al., 2016; BARROS, 2016; PERAZZO, 2016; FLORES-OLIVARES et al., 2015; SILVA et al., 2015; ROSSIGNOLI; BENITO; HERRERO, 2015; SANTOS, 2015; ANSELMO, 2014; PORTO, 2014; PEREIRA, 2013; SILVA et al., 2011; PRESTES, 2011; SILVA, 2011; LUZ et al., 2010; JENKINS; ROWN; RUTTERFORD, 2009; SCHACHER et al., 2008). Não obstante, as abordagens contemplam a análise de relações entre estes sujeitos e diferentes categorias de produtos.

A este respeito, as tecnologias assistivas destacam-se como a categoria predominante nos estudos encontrados (MERINO et al., 2017; PERAZZO, 2016; ROSSIGNOLI; BENITO; HERRERO, 2015; PORTO, 2014; SILVA et al., 2011; PRESTES, 2011; SILVA, 2011; LUZ et al., 2010). Ainda, apresentamse as categorias de peças de vestuário (SANTOS, 2015; ANSELMO, 2014; SCHACHER et al., 2008), equipamentos esportivos (PUŠNIK; ČUK; HADŽIČ, 2017; DOTTI et al., 2016), peças de mobiliário (SALES et al., 2017; FLORES-OLIVARES et al., 2015; PEREIRA, 2013) embalagens (BARROS et al., 2016; BARROS, 2016; SILVA et al., 2015) e ferramentas (TIRLONI et al., 2017). Além disso, um dos estudos apresenta experimentos com produtos intangíveis (JENKINS; ROWN; RUTTERFORD, 2009).

No que tange as categorias de tecnologias assistivas, destacam-se os estudos de Merino et al. (2017), Luz et al. (2010) e Porto (2014), que analisam órteses e próteses. Merino et al. (2017) aplicam a instrumentação integrada (termografia infravermelha, eletromiografia, dinamometria e captura de movimentos) para o desenvolvimento de produtos de tecnologia assistiva - órteses para pacientes de com deficiências múltiplas, doenças psiquiátricas e mobilidade reduzida. Os autores propõem um protocolo de coleta que atende às necessidades dos pesquisadores e respeita 
as limitações do usuário e concluem que os dados termográficos permitem a compreensão do estado muscular dos usuários, além de auxiliar na validação dos produtos desenvolvidos, aproximando as equipes de projeto das necessidades reais dos usuários.

$\mathrm{Na}$ mesma categoria de produto, Porto (2014) propõe um método de investigação quantitativa e qualitativa de órteses de neoprene com ênfase no conforto térmico, aplicando forros de diferentes tecidos e utilizando o neoprene liso e o perfurado. Não obstante, Luz et al. (2010) utilizam a termografia infravermelha para verificar a adaptação de uma prótese híbrida à extremidade superior de um sujeito com o braço amputado. A figura 4 apresenta a imagem térmica da região do tórax ao longo do tirante de fixação da prótese, antes e depois do equilíbrio térmico, indicando as temperaturas mais elevadas, como a da região da axila homolateral ao membro íntegro, coincidindo com a região relatada de maior desconforto.

Figura 4 - Imagem termográfica antes e depois do uso da prótese híbrida.

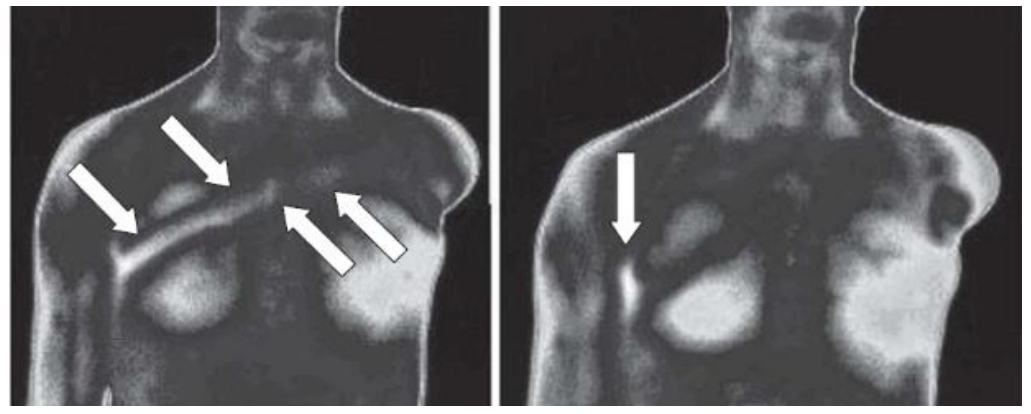

Fonte: Luz et al. (2010).

Já os trabalhos de Perazzo (2016), Prestes (2011), Silva (2011), Silva et al. (2011) e Rossignoli, Benito e Herrero (2015) relacionam-se a cadeiras de rodas, visando analisar o conforto de seus usuários. Perazzo (2016) dedica-se a avaliar a distribuição de pressão (assento e encosto) relacionada à variação do ângulo de tilt em cadeira de rodas para contribuir na prescrição de sistemas de adequação postural na posição sentada. Os sujeitos desta pesquisa possuíam idade entre 04 e 14 anos e diagnóstico de Paralisia Cerebral.

Prestes (2011), por sua vez, propõe novas rotas tecnológicas para a fabricação de assentos e encostos personalizados para pessoas deficientes e usuários cadeira de rodas. Para tanto, utilizase de ferramentas da digitalização tridimensional, da usinagem CNC e da termografia infravermelha para realizar medidas e analisar os assentos e encostos personalizados fabricados a partir de dados antropométricos individualizados. Os protótipos foram projetados, fabricados e testados.

O estudo de Silva (2011) visa disponibilizar uma alternativa tecnológica e economicamente viável para a fabricação de assentos personalizados para cadeiras de rodas. Para tanto, apresenta uma metodologia para a fabricação destes produtos, identificando parâmetros, materiais e ferramentas necessárias para o projeto e a execução (digitalização tridimensional e da usinagem CNC). Silva et al. (2011) estabelecem requisitos para o projeto destas almofadas personalizadas, utilizando o escâner 3D para reprodução das características do sujeito e a termografia infravermelha e os sensores de pressão para o mapeamento e comparação da distribuição da temperatura e da pressão dos assentos, conforme figura 5. O termograma do assento customizado (B) demonstra uma distribuição mais homogênea quando comparado ao assento padrão $(A)$, que atinge a 
temperatura 29,24드 após o uso, 3,31으 maior que o B. Isso demonstra um comportamento térmico mais regular e equilibrado do assento customizado, que resulta em um maior conforto ao usuário.

Figura 5 - Análise termográfica do assento padrão (A) e do assento customizado (B).
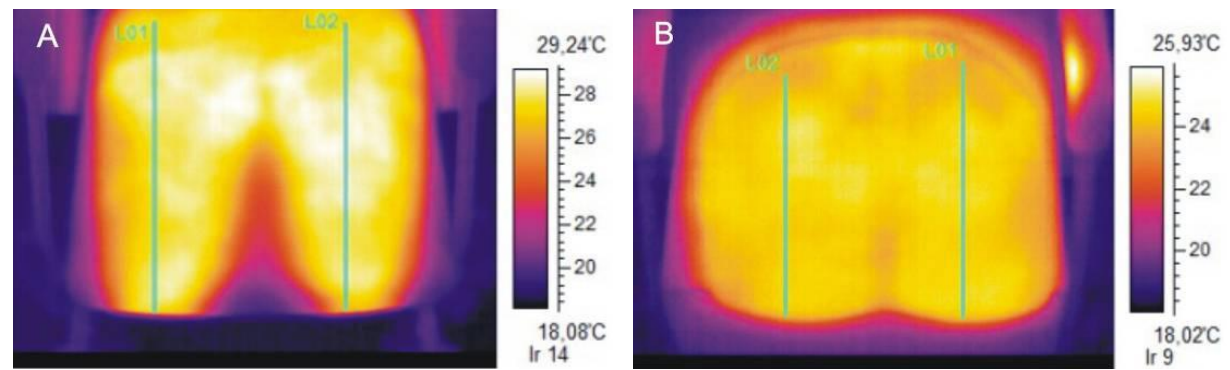

Fonte: Silva et al. (2011).

Rossignoli, Benito e Herrero (2015) verificam a confiabilidade da termografia infravermelha em usuários de cadeiras de rodas, utilizando-a como técnica não invasiva e sem risco para detectar a radiação térmica natural emitida pela pele humana e para permitir interpretações subsequentes das distribuições de temperatura. Os sujeitos possuíam paraplegia, tetraplegia e outras deficiências (amputação, esclerose múltipla e poliomielite).

Ainda a este respeito, destaca-se que nos estudos de tecnologias assistivas, são predominantes as avaliações de sujeitos com algum tipo de incapacidade física e/ou psíquica (MERINO et al., 2017; PERAZZO, 2016; ROSSIGNOLI; BENITO; HERRERO, 2015; PORTO, 2014; SILVA et al., 2011; PRESTES, 2011; SILVA, 2011; LUZ et al., 2010). Neste sentido, a termografia infravermelha é utilizada para avaliar a interação dos produtos com os usuários, visando maior conforto, eficiência e segurança. Em suma, em relação às tecnologias assistivas analisadas nos estudos, destacam-se produtos como as cadeiras de rodas (analisadas em cinco estudos), as órteses (analisadas em dois estudos) e as próteses (um estudo), conforme figura 6.

Figura 6 - Produtos analisados nos estudos.

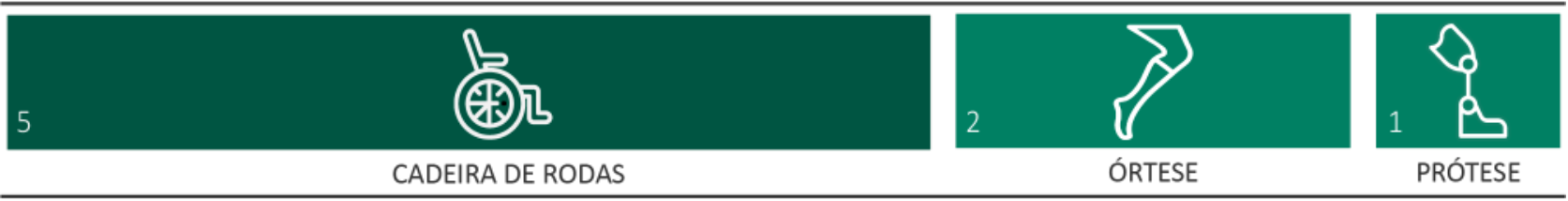

Fonte: Elaborado pela autora.

Nos trabalhos relacionados à vestuário, os produtos analisados foram roupas e calçados (SANTOS, 2015; ANSELMO, 2014; SCHACHER et al., 2008). Santos (2015) buscou determinar os efeitos de diferentes tipos de modelagem sobre a temperatura corporal, a transpiração e o conforto térmico percebido por usuários de camisetas, que foram fabricadas em tecidos com propriedades de termo regulação.

Também relacionado a peças de vestuário, Schacher et al. (2008) utilizaram a termografia infravermelha para verificar a eficiência de dois protótipos de produtos (vestimenta para dentistas), visando identificar a alternativa que proporcione maior proteção, segurança e conforto aos 
usuários. Ambos os trabalhos geram requisitos e especificações para o desenvolvimento de novos projetos de produtos. Já Anselmo (2014) investigou sapatilhas femininas de uso diário por meio da comparação de cinco diferentes forros. Neste estudo, a autora relacionou dados quantitativos e qualitativos relacionados ao conforto térmico percebido pelas usuárias.

Quanto aos equipamentos esportivos, foram encontrados os trabalhos de Pušnik, Čuk e Hadžič (2017) e Dotti et al (2016), que verificam a eficiência dos produtos quanto ao conforto oferecido ao usuário durante o uso. Pušnik, Čuk e Hadžič (2017) verificam a mudança de temperatura da pele das mãos durante o uso de anéis dinâmicos (tradicional e anatômico), para determinar qual possui um design mais confortável. Esse trabalho também associa as características morfológicas, a força de preensão com a mudança de temperatura da palma das mãos. Dotti et al. (2016) investigam as performances (em termos de gerenciamento de calor e de umidade) de três protetores de costas comerciais, conforme figura 7. O objetivo do estudo consistiu na identificação de parâmetros de projeto que afetam o conforto térmico durante atividades esportivas de inverno.

Figura 7 - Imagens térmicas das costas de um voluntário em momentos diferentes do teste.

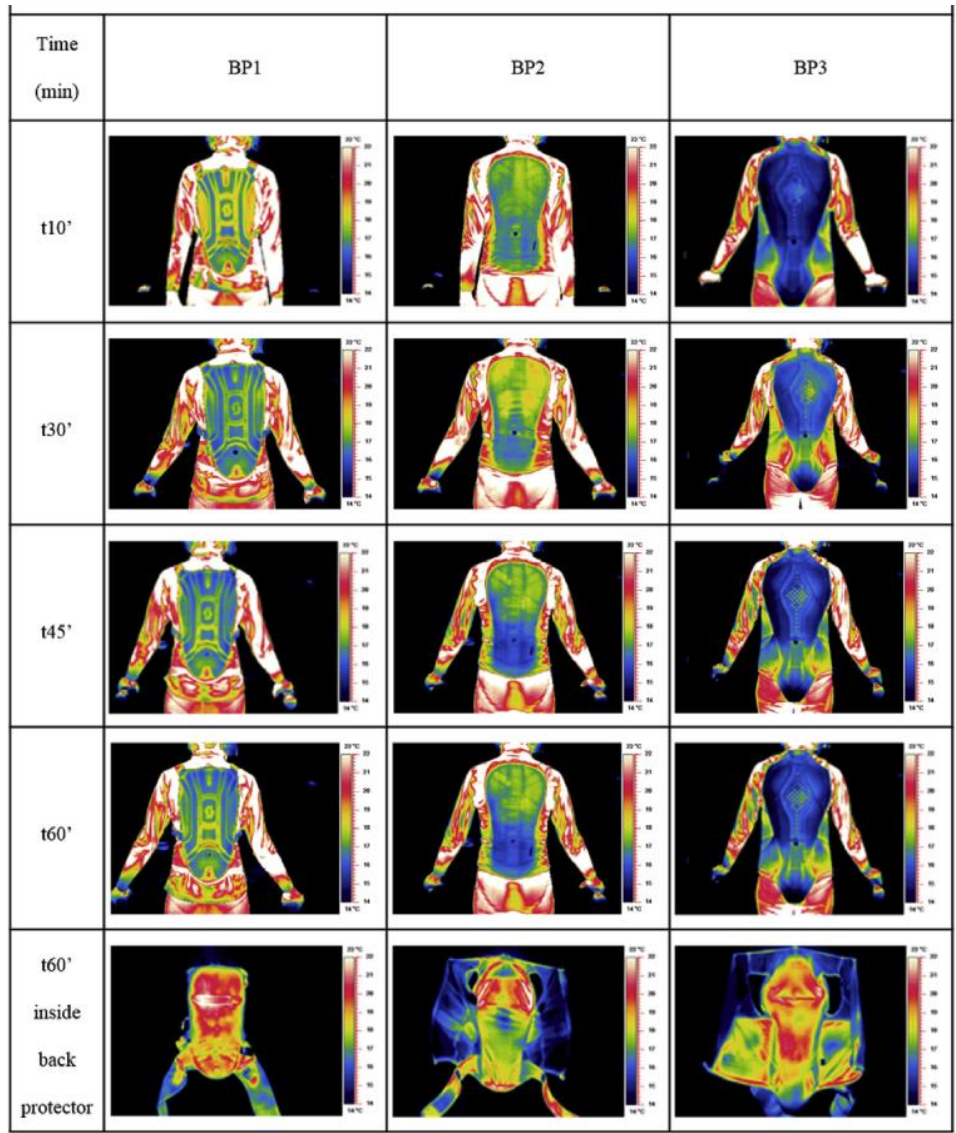

Fonte: Dotti et al. (2016).

Nos trabalhos sobre peças de mobiliário (SALES et al., 2017; FLORES-OLIVARES et al., 2015; PEREIRA, 2013) foram avaliadas cadeiras escolares e bancos para bateristas. Flores-Olivares et al. (2015) desenvolveram um protocolo para monitorar a temperatura da pele em um baterista profissional utilizando diferentes tipos de banco. A análises concentraram-se na região inferior das costas, devido aos relatos de desconforto dos usuários. 
Já Sales et al. (2017) avaliaram o comportamento térmico de assentos e encostos de oito cadeiras escolares de diferentes materiais, após seu uso, analisando a mudança de temperatura corporal do usuário após o uso dos produtos. Perreira (2013), coautor deste artigo, investigou o aquecimento e resfriamento (conforto térmico) nos assentos após um voluntário ficar sentado quinze minutos sobre eles. Os resultados deste estudo mostram que a temperatura se comporta de maneira semelhante, que o tempo de quinze a vinte minutos é o suficiente para que a temperatura se estabilize e que o resfriamento é maior nos cinco primeiros minutos. Assim, concluiu que a termografia pode contribuir para subsidiar o estudo do conforto térmico do assento de cadeiras, por se mostrar capaz de identificar a influência da temperatura em assentos fabricados com materiais diversos.

Os trabalhos de Barros et al. (2016), Barros (2016) e Silva et al. (2015) avaliaram a abertura de embalagens - garrafas PET de refrigerante - sob diferentes aspectos. Silva et al. (2015) avaliaram as variáveis de esforço e força de torque e as alterações térmicas das mãos dos indivíduos antes e após as tentativas de abertura de dois modelos de garrafas de PET com distintos modelos de tampa.

Ainda acerca de embalagens PET de refrigerantes, Barros (2016) realizou uma avaliação de usabilidade durante a manipulação manual destas embalagens, comparando a experiência relatada pelo usuário e a mensuradas por meio do rastreamento ocular, da termografia infravermelha, do eletroencefalograma e outras medidas subjetivas. Barros et al. (2016) apresentam um recorte desta pesquisa, investigando a interação usuário-produto (usabilidade) da abertura de garrafas PET por meio da termografia. Os resultados demonstram que a tecnologia foi eficiente na identificação de mudanças metabólicas, mas insuficiente para detectar expressões emocionais relatadas.

Não obstante, o trabalho de Jenkins, Rown e Rutterford (2009) trabalha com a combinação da termografia infravermelha, do Eletroencefalograma e de medidas subjetivas para avaliar interações de usuários com produtos. Os autores buscaram estabelecer se é possível usar a termografia infravermelha para medir mudanças no estado afetivo, correlacionando-a com o eletroencefalograma frontal (EEG) e o auto relatório afetivo, que são métodos validados para medir essas circunstâncias. No entanto, devido às limitações experimentais do EEG, especificamente a interferência criada pelo movimento físico do sujeito, optou-se por restringir as variáveis da interação instrumental e focar na interação não-física, estimulando o pensamento sobre o uso dos produtos para a execução de uma tarefa.

Por fim, Tirloni et al. (2017) visaram avaliar a temperatura das mãos dos trabalhadores de matadouros e sua relação com a sensação térmica das mãos e o uso de uma ferramenta de corte. Em síntese, a figura 8 apresenta as categorias de produtos analisados nos estudos.

Figura 8 - Categorias de produtos analisados nos estudos.

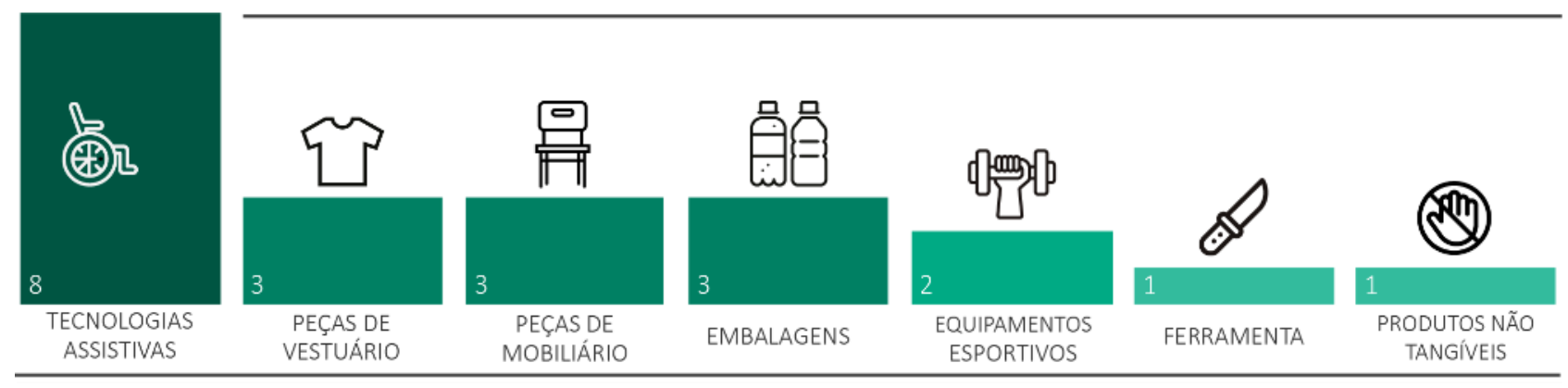

Fonte: Elaborado pela autora. 
Dentre os 21 resultados, 15 destacam-se os trabalhos que propõe a verificação e comparação de produtos (SALES et al., 2017; PUŠNIK; ČUK; HADŽIČ, 2017; BARROS et al., 2016; DOTTI et al., 2016; BARROS, 2016; FLORES-OLIVARES et al., 2015; SILVA et al., 2015; SANTOS, 2015; ANSELMO, 2014; PORTO, 2014; PEREIRA, 2013; SILVA et al., 2011; PRESTES, 2011; SILVA, 2011; SCHACHER et al., 2008). Estes estudos concentram-se na identificação das alternativas de produtos mais adequadas às necessidades dos projetos, utilizando a termografia infravermelha e outros instrumentos capazes de fornecer dados quantitativos e objetivos.

A este respeito, dos 21 estudos, 11 utilizam a termografia juntamente a outros métodos, como a captura de movimentos por sensores inerciais, eletromiografia de superfície, medidores de pressão e torque, dinamometria, eletroencefalograma, escâner 3D, usinagem CNC, sensores de temperatura e umidade, entre outros (MERINO et al., 2017; DOTTI et al., 2016; BARROS, 2016; PERAZZO, 2016; SILVA et al., 2015; SANTOS, 2015; ANSELMO, 2014; SILVA et al., 2011; PRESTES, 2011; SILVA, 2011; JENKINS; ROWN; RUTTERFORD, 2009).

\section{Conclusão}

Por meio deste estudo, foi possível demonstrar como a termografia infravermelha vem sendo aplicada no desenvolvimento de produtos, destacando as diferentes formas de abordagem, bem como as categorias dos produtos analisados. Para tanto, os procedimentos metodológicos utilizados se mostraram adequados e satisfatórios para o alcance do objetivo proposto.

Os resultados apontam que a aplicação da termografia infravermelha acontece em distintos momentos do desenvolvimento de projetos, como no levantamento de dados iniciais, na verificação de produtos existentes e na avaliação de alternativas de projeto. Os dados quantificáveis e objetivos gerados por meio desta tecnologia auxiliam na análise das relações de usabilidade, conforto e desconforto durante a interação entre usuários/sujeitos e produtos.

A termografia infravermelha oferece dados térmicos precisos, que comumente são associados à dados subjetivos e/ou fornecidos por outras tecnologias, possibilitando análises mais aprofundadas sobre os objetos de estudo e suas interações. Além disso, as análises comparativas também são recorrentes nos estudos levantados, possibilitando a avaliação de diferentes tipos e alternativas de produtos. Esta avaliação permite a escolha das soluções mais eficientes, confortáveis, seguras e adequadas às necessidades do projeto, ou seja, auxilia os profissionais na tomada de decisões no processo de desenvolvimento de produtos.

Além disso, os resultados apontam a aplicação da termografia infravermelha no desenvolvimento de produtos de distintas categorias, destacando as tecnologias assistivas, seguidas pelas peças de vestuário, equipamentos esportivos, peças de mobiliário, embalagens e ferramentas. As tecnologias assistivas denotam a categoria de produtos mais analisada por meio da termografia infravermelha, em oito dos vinte e um estudos avaliados, sendo representadas por produtos como as cadeiras de rodas (em 5 estudos), as órteses (em 2 estudos) e as próteses (em 1 estudo). Deste modo, estes estudos demonstram a efetividade da tecnologia em projetos envolvendo sujeitos com limitações físicas e psíquicas, devido a sua capacidade de detectar e quantificar temperaturas de maneira não invasiva, evitando interferências e possibilitando a obtenção de dados precisos mesmo diante de restrições comunicacionais. De modo geral, os resultados demonstram diversas possibilidades para a aplicação da termografia infravermelha no campo do design, no qual a tecnologia poderia ser utilizada de maneira mais recorrente e ampla. 
Contudo, percebem-se lacunas e oportunidades de estudos sobre a aplicação da termografia infravermelha no desenvolvimento de produtos. Como lacunas, nota-se a escassez de estudos sobre o tema, bem como a limitação das categorias de produtos analisados. Porém, como futuros estudos, pretende-se dar continuidade a análise de conteúdo dos estudos levantados, a fim de identificar os protocolos de coleta de dados e os tipos de análise adotados.

\section{Referências}

ANSELMO, Taiza K. Parâmetros para o Desenvolvimento de Sapatilhas Femininas de uso diário com Ênfase no Conforto Térmico e Percepção da Usuária. 2014. 121 f. Dissertação (Mestrado em Design) - Universidade Estadual de Santa Catarina. Programa de Pós-Graduação em Design. Florianópolis, 2014. Disponível em: <http://tede.udesc.br/handle/handle/2025>. Acesso em: 15 out. 2017.

BARROS, Rafaela Q. de. Aplicação da neuroergonomia, rastreamento ocular e termografia por infravermelho na avaliação de produto de consumo: um estudo de usabilidade. 2016. Dissertação (Mestrado). Programa de Pós-Graduação em Design da Universidade Federal de Pernambuco, Pernambuco. Disponível em: < http://repositorio.ufpe.br/handle/123456789/17912>. Acesso em: 15 out. 2017.

BARROS, Rafaela Q. et al. Using Digital Thermography to Analyse the Product User's Affective Experience of a Product. Advances In Ergonomics In Design, [s.I.], p.97-107, 2016. Springer International Publishing.

BEST, Kathryn. Fundamentos da gestão do design. Porto Alegre: Bookman, 2012.

BRIOSCHI, Marcos L.; MACEDO, José F; MACEDO, Rodrigo de A. C. Termometria cutânea: novos conceitos. Jornal Vascular Brasileiro, v. 2, p. 151-60, 2003.

DOTTI, Francesca et al. Thermo-physiological comfort of soft-shell back protectors under controlled environmental conditions. Applied Ergonomics, [s.I.], v. 56, p.144-152, set. 2016. Elsevier BV.

FLIR. 3 principais aplicações da análise termográfica. (2016). Disponível em: <http://www.flir.com.br/home/news/details/?ID=80767>. Acesso em: 15 out. 2017.

FLORES-OLIVARES, Blanca del Carmen et al. Preliminary Study on the Evaluation of Musculoskeletal Risks through Infrared Thermography for Drummers. Procedia Manufacturing, [s.I.], v. 3, p. 44154420, 2015. Elsevier BV.

GABRIEL, Joaquim et al. Termografia: imagem médica e síndromes dolorosas. Lisboa: Lidel, 2016.

GIL, Antonio Carlos. Métodos e técnicas de pesquisa social. 6. ed. - São Paulo: Atlas, 2008.

GOMES FILHO, João. Design do objeto: bases conceituais. São Paulo: Escrituras Editora, 2006.

GREENHALGH, Trisha. How to read a paper: Papers that summarise other papers (systematic reviews and meta-analyses). Bmj, [s.I.], v. 315, n. 7109, p.672-675, 13 set. 1997. BMJ.

HOLST, Gerald C. Common sense approach to thermal imaging. Washington, DC, USA: SPIE Optical Engineering Press, 2000.

JENKINS, Sean, BROWN, Raymond; RUTTENFORD, Nail. Comparing thermographic, EEG, and subjective measures of affective experience during simulated product interactions. International Journal of Design. Taipei, v. 3, n. 2, p.53-65, ago. 2009. 
KOLLER, Sílvia H.; DE PAULA COUTO, Maria Clara P.; VON HOHENDORFF, Jean. Manual de produção científica. Porto Alegre: Penso Editora, 2014.

LOBACH, Bernd. Design Industrial: bases para a configuração dos produtos industriais. São Paulo: Edgar Blucher, 2001.

LUZ, Soraia C. T. da et al. Adaptação à prótese híbrida de extremidade superior: estudo termográfico de um caso. Fisioterapia e Pesquisa, [s.I.], v. 17, n. 2, p.173-177, jun. 2010. FapUNIFESP (SciELO).

MERINO, Eugenio et al. Implementation of Integrated Instrumentation in Assistive Technology. Advances In Ergonomics In Design, [s.I.], p.549-560, 24 jun. 2017. Springer International Publishing.

MOBLEY, R. Keith. An introduction to predictive maintenance. 1. ed. Amsterdam:ButterworthHeinemann, 2002.

MORRIS, Richard. Fundamentos de design de produto. Porto Alegre: Bookman Editora, 2010.

PEREIRA, Romeu R. Características térmicas de assento de cadeiras escolares por termografia. 2013. Dissertação (Mestrado). Escola de Design, Programa de Pós-Graduação em Design da Universidade do Estado de Minas Gerais, Belo Horizonte, 91. 2013. Disponível em: <http://www.ppgd.uemg.br/wp-content/uploads/2013/10/Romeu_Rodrigues_Pereira.pdf>. Acesso em: 15 out. 2017.

PERAZZO, A. L. Tecnologia assistiva: a influência do ângulo do tilt sobre as pressões em assentos de cadeiras de rodas. Dissertação de Mestrado (Mestre em Design) - Escola de Engenharia, Universidade Federal do Rio Grande do Sul, Porto Alegre, p. 115. 2016. Disponível em: < http://hdl.handle.net/10183/143979 >. Acesso em: 15 out. 2017.

PORTO, Maetê da Costa. Método de Avaliação de Conforto Térmico em Órteses Esportivas de Joelho. 2014. Projeto (Mestrado em Design) - Universidade do estado de Santa Catarina. Programa de Pós-Graduação em Design, Florianópolis, p. 128. 2014. Disponível em: < http://tede.udesc.br/handle/handle/2028>. Acesso em: 15 out. 2017.

PRESTES, Rafael C. Tecnologia assistiva: atributos de design de produto para adequação postural personalizada na posição sentada. 2011. (Mestrado em Design) - Universidade do estado do Rio Grande do Sul. Programa de Pós-Graduação em Design, p.97, 2011. Disponível em: < http://hdl.handle.net/10183/36038>. Acesso em: 15 out. 2017.

PUŠNIK, Igor; ČUK, Ivan; HADŽIČ, Vedran. Influence of new anatomic ring design on palm skin temperature. Science of Gymnastics Journal, v. 9, n. 1, p. 61-70, 2017.

ROSSIGNOLI, Isabel; BENITO, Pedro J; HERRERO, Azael J. Reliability of infrared thermography in skin temperature evaluation of wheelchair users. Spinal Cord, [s.I.], v. 53, n. 3, p.243-248, 25 nov. 2014. Springer Nature.

SALES, Rosemary B. C. et al. Thermal comfort of seats as visualized by infrared thermography. Applied Ergonomics, [s.I.], v. 62, p.142-149, jul. 2017. Elsevier BV.

SAMPIERI, Roberto H.; COLLADO, Carlos F.; LUCIO, M. P. B. Métodos de Pesquisa. 5. ed. Porto Alegre: Penso, 2013.

SANTOS, Roberta M. V. Relação entre design da modelagem e aplicação de materiais de mudança de fase no vestuário: uma análise do conforto térmico com base nos fatores humanos. 2015. 143 
f. Dissertação (Mestrado em Design) - Universidade Estadual de Santa Catarina. Programa de PósGraduação em Design. Florianópolis, 2015. Disponível em: < http://tede.udesc.br/handle/handle/2031>. Acesso em: 15 out. 2017.

SCHACHER, M., et al. Improvement of dentist gowns - new constraints and new risks. International Journal of Clothing Science and Technology 21(4): 180-192, 2008.

SILVA, Danilo C. et al. Evaluation of Two PET Bottles Caps: An Exploratory Study. Procedia Manufacturing, [s.l.], v. 3, p.6245-6252, 2015. Elsevier BV. http://dx.doi.org/10.1016/j.promfg.2015.07.768.

SILVA, Fábio P. Usinagem de espumas de poliuretano e digitalização tridimensional para fabricação de assentos personalizados para pessoas com deficiência. Tese (Doutorado em Engenharia) - Universidade Federal do Rio Grande do Sul. Programa de Pós-Graduação em Engenharia de Minas. Porto Alegre, 2011. Disponível em: <http://hdl.handle.net/10183/36040>. Acesso em: 15 out. 2017.

SILVA, Fabio P. et al. Design and milling manufacture of polyurethane custom contoured cushions for wheelchair users. The Australasian medical journal, v. 4, n. 9, p. 500, 2011.

SILVA, Júlio César R. P.; MIRA, Maria do R. G. Termografia: ferramenta auxiliar na pesquisa de materiais e no design de produtos. Libro de Actas - Systems \& Design: Beyond Processes and Thinking (IFDP - SD2016), [s.I.], p.377-391, 22 jun. 2016. Universitat Politècnica València. Disponível em: <http://ocs.editorial.upv.es/index.php/IFDP/IFDP/paper/viewFile/3651/2097>. Acesso em: 15 out. 2017.

SILVA, Júlio Cézar R. P. da; TARALLI, Cibele H.; MELZ, Simone P. M. Termograma: A imagem térmica como instrumento de diagnóstico rápido no design. In: Fourth International Conference on Integration of Design, Engineering and Management for innovation. Anais... Florianópolis, SC, Brasil: 2015. Disponível em: <http://janainaramos.com.br/idemi2015/anais/02/143332.pdf>. Acesso em: 15 out. 2017.

SILVA, Luiz A. da. Termografia: Princípios básicos e suas aplicações. 1. ed. São José dos Campos: Tecnolass Tecnologia, 2017.

SPECK, Giselle. M. et al. Processo de instrumentação integrada no desenvolvimento de projetos de Tecnologia Assistiva. In: $\mathbf{1 8}^{\circ}$ Congresso Brasileiro de Ergonomia (Abergo). Anais..., Belo Horizonte, MG, Brasil: 2016.

TIRLONI, Adriana S., et al. Thermographic evaluation of the hands of pig slaughterhouse workers exposed to cold temperatures. International Journal of Environmental Research and Public Health, v. 14, n. 8, 2017.

\section{Agradecimentos}

Agradecemos ao Programa de Pós-graduação em Design da UFSC (PPGD/UFSC), ao Núcleo de Gestão de Design e Laboratório de Design e Usabilidade (NGD-LDU/UFSC), à Rede de Pesquisa e Desenvolvimento em Tecnologia Assistiva (RPDTA), à Coordenação de Aperfeiçoamento de Pessoal de Nível Superior (CAPES), ao Conselho Nacional de Desenvolvimento Científico e Tecnológico (CNPq), e aos demais envolvidos. 